\title{
Robust topology design of periodic grating surfaces
}

Friis, Kasper Storgaard; Sigmund, Ole

Published in:

Optical Society of America. Journal B: Optical Physics

Link to article, DOI:

10.1364/JOSAB.29.002935

Publication date:

2012

Document Version

Publisher's PDF, also known as Version of record

Link back to DTU Orbit

Citation (APA):

Friis, K. S., \& Sigmund, O. (2012). Robust topology design of periodic grating surfaces. Optical Society of America. Journal B: Optical Physics, 29(10), 2935-2943. https://doi.org/10.1364/JOSAB.29.002935

\section{General rights}

Copyright and moral rights for the publications made accessible in the public portal are retained by the authors and/or other copyright owners and it is a condition of accessing publications that users recognise and abide by the legal requirements associated with these rights.

- Users may download and print one copy of any publication from the public portal for the purpose of private study or research.

- You may not further distribute the material or use it for any profit-making activity or commercial gain

- You may freely distribute the URL identifying the publication in the public portal

If you believe that this document breaches copyright please contact us providing details, and we will remove access to the work immediately and investigate your claim. 


\title{
Robust topology design of periodic grating surfaces
}

\author{
Kasper Storgaard Friis and Ole Sigmund* \\ Department of Mechanical Engineering, Technical University of Denmark, Niels Koppels Alle, Building 404-1, \\ Kongens Lyngby 2800, Denmark \\ ${ }^{*}$ Corresponding author: sigmund@mek.dtu.dk
}

Received June 4, 2012; revised August 6, 2012; accepted August 31, 2012;

posted September 5, 2012 (Doc. ID 169710); published September 28, 2012

\begin{abstract}
Modern nanoscale manufacturing techniques allow for a high degree of flexibility in designing surface microstructures and nanostructures. Injection molding of nanosized features allows for mass production of plastic components with a tailored nanostructure producing specific optical effects depending on the purpose. This work details the use of topology optimization for designing periodic polymer grating surfaces with complex optical properties. A method based on robust topology optimization is formulated for designing the nanostructure of plastic surfaces with extreme reflection or transmission properties. Topology optimization allows for free distribution of material but a mechanical constraint based on the fundamental free mechanical vibration frequency ensures connected structures. Several examples are given to illustrate the efficiency of the method. (c) 2012 Optical Society of America
\end{abstract} OCIS codes: $\quad 000.4430,050.2770,240.6700,330.7326$

\section{INTRODUCTION}

In many applications the need for specifying and controlling the optical properties of surfaces is of high importance. Applications include but are not limited to optical filters, sensors, holography, and diffraction gratings. As the applications become more and more advanced the requirements to the optical properties become increasingly complex. The problem of designing a surface with specific optical properties can therefore be extremely challenging. The fabrication of advanced nanostructured surfaces is made possible with modern nanotechnology, and hence engineers have an increasingly high degree of freedom to design complex surface structures [1-4].

The presented work is motivated by the developments in nanotechnology and injection molding improving the possibilities for achieving submicrometer features in the plastic surfaces sometimes directly from the casting process. This potentially allows for creating functional surfaces in plastic parts in large batch sizes. Properties such as antiwetting, hydrophobic and surfaces with tailored optical properties are features that could be utilized in industrial injection-molded plastic products. The focus of the present investigation is the modeling of the optical properties of nanostructured plastic surfaces and surface design optimization for achieving specific reflection and transmission characteristics. This work is a first step toward the goal of developing a methodology for designing three-dimensional (3D) surfaces displaying structural color properties imitating the wings of a butterflies and other animals [믁].

Topology optimization was originally developed as a method for designing optimal material distribution in elasticity but has recently been introduced to photonics for optimizing several different applications [8]. Some of the first attempts on using topology optimization for optimizing periodic gratinglike structures were done by Dobson $[\underline{9}, \underline{10}]$. The computational domain (the unit cell) is discretized using the finite element method and due to the periodicity of the structure the incoming and outgoing waves are modeled as plane wave expansions. For optimization a gradient descent minimization scheme is used and several examples are presented for plastic/air interfaces for both free material distributions [9] and shape-optimized gratings [10]. For the free material distribution approach the solutions all have problems reaching a discrete design (material being either air or plastic) and postprocessing of the designs are necessary. Shape designs are restricted to single interface designs and Dobson finds that constraints are required to regularize the design and avoid mesh-dependent solutions and increasingly smaller details in the optimized designs. He solves this by imposing a constraint on the integrated curvature of the grating profile. Fuchi et al. [11] have more recently presented a method based on the same ideas as in [9]; however, not restricted to optimize surfaces but rather considering a volume of dielectric. They interpolate the element material properties using the so-called SIMP scheme [12] and use a smoothing density filter with 1.5 element filter radius to avoid too small details and complex designs. Due to the filter the final designs have elements of intermediate densities at material transitions zones and binary designs are therefore achieved by postprocessing the solution.

The present work is centered on how smoothing filters of arbitrary size and a robust projection scheme can be employed in topology optimization to achieve mesh-independent and close-to binary solutions. The robust formulation ensures that the design is robust toward small variations in the design and manufacturing tolerances in a real production setting. Furthermore, the formulation allows for advanced combinations of desired transmittance/reflectance output characteristics by defining multiple objective functions or constraints. A detailed description of the numerical formulation is given as well as selected test cases demonstrating the method. Furthermore, it is considered how to facilitate manufacturable designs and avoid arbitrarily small or disconnected features by ensuring a minimum length scale in the designs through mechanical constraints in the optimization. 


\section{PROBLEM DESCRIPTION AND MODELING}

The problem of modeling a periodic surface structure can be restricted to only considering a single "supercell" of an airsurface interface. The two-dimensional (2D) computational domain for modeling a $y$-periodic surface is shown in Fig. 1 . The domain $\Omega$ consists of three regions: an air region $\Omega_{A}$, a surface region $\Omega_{D}$, and a bulk region $\Omega_{B}$, as indicated in Fig. 1 . The air and bulk regions have fixed material properties while material is distributed in the surface region to form the surface microstructure. The incoming wave enters the domain at boundary $\Gamma_{I}$ and the transmitted and reflected waves leave the domain through boundary $\Gamma_{O}$ and $\Gamma_{I}$, respectively. The modeling follows the procedure by Jensen et al. [13]. The problem can be modeled using the frequency-domain method based on a $2 \mathrm{D}$ model (plane polarization). This implies that the electromagnetic field in domains $\Omega_{A}, \Omega_{B}$, and $\Omega_{D}$ can be described by the 2D Helmholtz equation in Eq. (1):

$$
\nabla \cdot(A(\mathbf{x}) \nabla u(\mathbf{x}))+\omega^{2} B(\mathbf{x}) u(\mathbf{x})=0,
$$

where $\omega$ is the wave frequency $\left(\omega=c k_{0}\right), k_{0}$ the free-space wavenumber, $u(\mathbf{x})$ is the unknown spatial electric or magnetic field amplitude, and $A(\mathbf{x})$ and $B(\mathbf{x})$ are material coefficients depending on the polarization of the light. For $E_{z}$-polarization (only the $E$-field has an $z$ component) $A=1 / \mu_{r}$ and $B(\mathbf{x})=\epsilon_{r}(\mathbf{x}) c^{-2}$, and for $H_{z}$-polarization $A=1 / \epsilon_{r}(\mathbf{x})$ and $B(\mathbf{x})=\mu_{r} c^{-2}$, where $\mu_{r}$ is the relative permeability, $\epsilon_{r}(\mathbf{x})$ is the relative permittivity, and $c$ is the speed of light in vacuum. The incident field is given by

$$
u_{0}(\mathbf{x})=U \exp \left(-j \omega \sqrt{A^{-1} B} \hat{k} \cdot \mathbf{x}\right) .
$$

The factor $U$ is a scaling factor for the wave amplitude in the following set to unity, $\hat{k}=(\cos (\theta), \sin (\theta))$ is the wave directional unit vector specifying the direction of the incident wave, and $\mathbf{x}$ the spatial coordinates. The incident wave is specified on $\Gamma_{I}$ as given in Eq. (3) with the unit-normal vector $\hat{n}=(-1,0)$ according to Fig. $\underline{1}$ :

$$
\hat{n} \nabla u=j \omega \sqrt{A B}(1-\hat{n} \cdot \hat{k}) u_{0}=j \omega \sqrt{A B}(1+\cos (\theta)) u_{0} .
$$

The $y$-periodicity is enforced by Bloch-Floquet wave boundary conditions on $\Gamma_{a}$ and $\Gamma_{b}$. Based on the supercell in Fig. 1 the periodic boundary conditions can be formulated as

$$
u(x, d)=u(x, 0) \exp \left(-j \omega d \sin (\theta) \sqrt{B A^{-1}}\right) .
$$

It is noted that the Bloch-Floquet boundary condition depends on both the frequency and the incident angle of

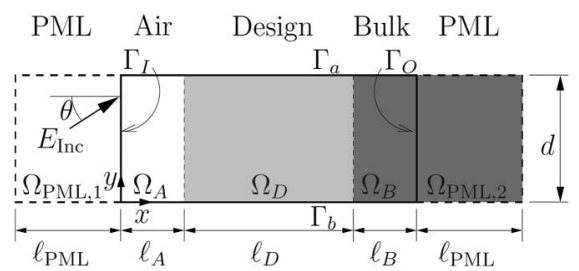

Fig. 1. Computational domain composed of a fixed air region $\Omega_{A}$, a fixed bulk region $\Omega_{B}$, and an active optimization region $\Omega_{D}$ where material is distributed to form the surface structure. the incoming wave, and the system matrix therefore also becomes a function of the frequency and incident angle. The domain is truncated using the standard method of perfectly matched layers (PMLs) [14] at the input and output boundaries for absorbing the waves. The governing equation in the $\Omega_{\mathrm{PML}}$ regions is

$$
\frac{\partial}{\partial x}\left(\frac{s_{y}}{s_{x}} A \frac{\partial u}{\partial x}\right)+\frac{\partial}{\partial y}\left(\frac{s_{x}}{s_{y}} A \frac{\partial u}{\partial y}\right)+\omega^{2} s_{x} s_{y} B u=0,
$$

where $s_{x}$ and $s_{y}$ are the spatial dependent complex damping factors in the $x$ and $y$ directions. The wave only has to be attenuated in the $x$ direction implying that $s_{y}=1$ and $s_{x}$ is given by

$$
s_{x}(x)=1-j \frac{\sigma(x)}{\omega \epsilon},
$$

where $\sigma(x)=\alpha\left|\left(x-x_{0}\right) / \ell_{\mathrm{PML}}\right|^{p}$ is the complex damping function, $\alpha$ and $p$ are the damping function parameters, and $x-x_{0}$ is the distance to the PML layer boundary. The material parameters $A$ and $B$ in the PML layer equals the fixed parameters in the domains $\Omega_{A}$ and $\Omega_{B}$ for the left and right PML regions, respectively. To solve Eq. (1) we write it in its weak form where $T$ is the so-called test function. By applying Greens first scalar identity and also considering the PML region and the input boundary condition given by Eqs. (5) and (3), respectively, the weak form of the problem becomes

$$
\begin{aligned}
\int_{\Omega}(A \nabla u) \cdot(\nabla T) \mathrm{d} \Omega & -\int_{\Omega} T \omega^{2} B u \mathrm{~d} \Omega \\
& +\int_{\Omega_{\mathrm{PML}}}\left(\frac{s_{y}}{s_{x}} A \frac{\partial u}{\partial x} \frac{\partial T}{\partial x}+\frac{s_{x}}{s_{y}} A \frac{\partial u}{\partial y} \frac{\partial T}{\partial y}\right) \mathrm{d} \Omega \\
& -\int_{\Omega_{\mathrm{PML}}} T \omega^{2} s_{x} s_{y} B u \mathrm{~d} \Omega \\
& =\int_{\Gamma_{I}} T j \omega \sqrt{A B}(1+\cos (\theta)) u_{0} \mathrm{~d} \Gamma .
\end{aligned}
$$

The domain is discretized and shape functions $\mathbf{N}(\mathbf{x})$ are used for interpolating the field $u=u(\mathbf{x})=\mathbf{N}(\mathbf{x}) \mathbf{u}$. If we furthermore choose the test function being identical to the shape function we obtain the system of equations

$$
\left(\mathbf{K}-\omega^{2} \mathbf{M}\right) \mathbf{u}=\mathbf{f},
$$

where $\mathbf{u}$ is a vector of discretized nodal values of the scalar field $u(\mathbf{x})$ and the finite element matrices $\mathbf{K}, \mathbf{M}$ and $\mathbf{f}$ are given by

$$
\begin{aligned}
\mathbf{K} & =\sum_{e \in \Omega} A_{e}\left(\mathbf{K}_{x}^{e}+\mathbf{K}_{y}^{e}\right)+\sum_{e \in \Omega_{\mathrm{PML}}} A_{e}\left[\left(\frac{s_{y}}{s_{x}}\right) \mathbf{K}_{x}^{e}+\left(\frac{s_{x}}{s_{y}}\right) \mathbf{K}_{y}^{e}\right], \\
\mathbf{K}_{x}^{e} & =\int \frac{\partial \mathbf{N}^{T}}{\partial x} \frac{\partial \mathbf{N}}{\partial x} \mathrm{~d} V, \quad \mathbf{K}_{y}^{e}=\int \frac{\partial \mathbf{N}^{T}}{\partial y} \frac{\partial \mathbf{N}}{\partial y} \mathrm{~d} V \\
\mathbf{M} & =\sum_{e \in \Omega} B_{e} \mathbf{M}^{e}+\sum_{e \in \Omega_{\mathrm{PML}}} B_{e}\left(s_{x} s_{y}\right)_{e} \mathbf{M}^{e}, \quad \mathbf{M}^{e}=\int \mathbf{N}^{T} \mathbf{N} \mathrm{d} V, \\
\mathbf{f} & =j \omega(1+\cos (\theta)) \sum_{e \in \Gamma_{I}} \sqrt{A_{e} B_{e}} \mathbf{f}^{e}, \quad \mathbf{f}^{e}=\oint \mathbf{N}^{T} u_{0} \mathrm{~d} S .
\end{aligned}
$$

In Eq. (9) $A_{e}, B_{e}, s_{x}$, and $s_{y}$ are assumed element-wise constants. The integration of the element force term $\mathbf{f}^{e}$ can be 
done analytically but becomes lengthy and is therefore not shown. The Bloch-Floquet periodic boundary conditions are enforced by direct penalization of the system matrix.

\section{TOPOLOGY OPTIMIZATION PROBLEM}

\section{A. Material Interpolation}

We wish to apply a gradient-based algorithm and therefore use continuous design variables to control the distribution of air and dielectric in the design domain [12]. In $\Omega_{D}$ each element is assigned a design variable $\rho_{e}$ where

$$
0 \leq \rho_{e} \leq 1, \quad e \in \Omega_{D}
$$

The elemental material parameters $A_{e}$ and $B_{e}$ are then interpolated using the so-called SIMP scheme [12].

$$
A_{e}=A_{1}+\rho_{e}^{p}\left(A_{2}-A_{1}\right), \quad B_{e}=B_{1}+\rho_{e}^{p}\left(B_{2}-B 1_{1}\right),
$$

where $p$ is the penalization parameter. The goal of the optimization routine is to find the values of $\rho_{e}$ that optimize the chosen objective function and ensure discrete 0-1 designs to have a well-defined final structure. In structural optimization it is often necessary to penalize the optimization $(p>1)$ in order to improve convergence toward discrete designs. For certain optical problems this penalization is often not strictly needed to approach discrete solutions $[13,15]$. In this work $p$ has been set to 1 ; however, the robust optimization scheme indirectly ensures discrete designs. The design variables are smoothed by filtering and subsequently projected into the physical densities representing the actual physical design. This is explained in detail in Subsection 3.C.

\section{B. Objective Function}

The goal can be defined as either optimizing the transmittance or the reflectance of the surface given by

$$
T=\frac{P_{T}}{P_{0}} \quad R=\frac{P_{R}}{P_{0}},
$$

where $P_{T}$ is the transmitted power through the surface, $P_{R}$ is reflected power from the surface, and $P_{0}$ is the power flow of the incident wave. As the domain is periodic, the total transmittance or reflectance can be measured by considering the total power in the supercell. If $P_{\text {in }}$ designates the power entering the domain from the left boundary and $P_{\text {out }}$ the power leaving the domain at the right boundary we have that $P_{\text {out }}=P_{T}$ and $P_{\text {in }}=P_{0}-P_{R}$, implying that we can formulate the following objective functions:

$$
\Phi_{0}^{T}=\frac{P_{\text {out }}}{P_{0}} \quad \Phi_{0}^{R}=\frac{P_{0}-P_{\text {in }}}{P_{0}}=1-\frac{P_{\text {in }}}{P_{0}} .
$$

The total power-flow can be calculated from the timeaveraged Poynting vector given as

$$
\mathbf{V}(\mathbf{x})=\frac{1}{2 \omega} \mathfrak{R}(j C \bar{u} \nabla u),
$$

where $C$ equals $1 / \mu_{0} \mu_{r}$ and $1 / \epsilon_{0} \epsilon_{r}$ for $E_{z}$ and $H_{z}$ polarization, respectively. As the domain is infinite in the $y$-direction the power leaving or entering the domain is found as the integral of the $x$-component $P_{x}$ of the power-flow across a vertical line in the domain. Element-wise $P_{x}$ is calculated as

$$
\left(P_{x}\right)_{e}=\frac{C_{e}}{2 \omega} \Re\left(i\left(\mathbf{u}_{e}\right)^{T} \mathbf{Q}^{e} \overline{\mathbf{u}}_{e}\right),
$$

where $\overline{\mathbf{u}}^{e}$ denotes the complex conjugate of the element nodal values and $\mathbf{Q}^{e}$ is defined as

$$
\mathbf{Q}^{e}=\left.\int \frac{\partial \mathbf{N}^{T}}{\partial x} \mathbf{N d} y\right|_{x=x_{\Gamma}}
$$

This implies that the input and exit powers can be calculated by

$$
P_{\text {in }}=\sum_{e \in e_{\text {in }}}\left(P_{x}\right)_{e} \quad P_{\text {out }}=\sum_{e \in e_{\text {out }}}\left(P_{x}\right)_{e}
$$

where $e_{\text {in }}$ and $e_{\text {out }}$ are the elements at the input $\left(x_{\Gamma}=0\right)$ and exit boundary $\left(x_{\Gamma}=\ell_{A}+\ell_{B}+\ell_{D}\right)$, respectively.

\section{Sensitivities and Filtering}

The sensitivities are found using the adjoint method [12]. In our problem the right-hand side of the finite element (FE) equation [Eq. (8)] $\mathbf{f}$ is independent of the design variables and the design and output domains do not overlap. From [16] we therefore have that the sensitivities of the objective function $\Phi$ with respect to design changes can be written as

$$
\frac{d \Phi}{d \rho_{i}}=2 \Re\left(\lambda^{T} \frac{\partial \mathbf{S}}{\partial \rho_{i}} \mathbf{u}\right),
$$

where $\mathbf{S}=\mathbf{K}-\omega \mathbf{M}$ is the system matrix and adjoint vector $\lambda$ is the solution to the adjoint equation

$$
\mathbf{S}^{T} \lambda=-\frac{1}{2}\left(\frac{\partial \Phi_{0}}{\partial \mathbf{u}_{R}}-i \frac{\partial \Phi_{0}}{\partial \mathbf{u}_{I}}\right) .
$$

Assuming that all material is lossless the material parameter $C_{e}$ in Eq. (15) is real and the right-hand side of Eq. (19) can be found as

$$
\mathbf{S}^{T} \lambda=\sum_{e \in e_{\mathrm{in}}} \frac{i C_{e}}{4 \omega}\left(\mathbf{Q}^{e}-\left(\mathbf{Q}^{e}\right)^{T}\right)^{T} \overline{\mathbf{u}}_{e}
$$

We note that the system matrix in Eq. (20) is transposed implying that we can not reuse the factorization of $\mathbf{S}$ from solving the actual FE-problem in Eq. 8 .

Problems such as mesh dependency and element-size features in the optimized solutions are solved by introducing filtering of the design variables that smoothes the variables and ensures regularization of the design problem. However, simple filtering leads to gray transitions zones of intermediate physical design variables between 0 and 1 [17]. For some problems (e.g., statical mechanical) the transition zones have negligible influence on the performance and simple cutoff postprocessing will provide good interpretation for a final discrete design. For problems with more complex physics and/or objective functions the transition zones may be of large importance for the design performance implying that simple cutoff postprocessing could give a wrong physical interpretation of the design. Therefore, recently proposed projection techniques $[17,18]$ project the filtered design into the discrete $1 / 0$ space during the optimization and thereby eliminate the need 
for postprocessing of designs. In the following the original design variables are denoted $\rho_{i}$, the filtered design variables $\tilde{\rho}_{i}$, and the projected physical density $\bar{\rho}_{i}$. The filtered variable for cell $i$ is calculated as

$$
\tilde{\rho}_{i}=\frac{\sum_{j \in \mathbb{N}_{e, i}} w\left(\mathbf{x}_{j}\right) v_{j} \rho_{j}}{\sum_{j \in \mathbb{N}_{e, i}} w\left(\mathbf{x}_{j}\right) v_{j}},
$$

where $\mathbb{N}_{e, i}$ is the set of neighborhood elements within the filter domain of element $i, v_{j}$ is the volume of element $j$, and $w\left(\mathbf{x}_{j}\right)$ is the weighting function

$$
w\left(\mathbf{x}_{j}\right)=R-\left|\mathbf{x}_{j}-\mathbf{x}_{i}\right|,
$$

with filter radius $R$ and $\mathbf{x}$ containing the element central coordinates. The projection function is given by the smooth function [19]:

$$
\bar{\rho}_{i}=\frac{\tanh (\beta \eta)+\tanh \left(\beta\left(\tilde{\rho}_{i}-\eta\right)\right)}{\tanh (\beta \eta)+\tanh (\beta(1-\eta))} .
$$

In (23) $\beta$ is a parameter that controls the steepness of the projection function and $\eta$ is the threshold value below which filtered densities $\tilde{\rho}_{i}$ are projected to 0 and above to 1 . In order to ensure a smooth optimization a beta-continuation scheme [18] is applied to gradually approach the $0 / 1$ Heaviside step function given by Eq. (23) as the optimization progresses. The $\beta$ parameter is increased every 50 iteration or if the change in design variables drops below a given threshold. Due to the filtering and projection of the design variables, the design sensitivities with respect to the original design variables are calculated by the chain rule

$$
\frac{\partial \Phi}{\partial \rho_{j}}=\sum_{i \in \mathbb{N}_{e j}} \frac{\partial \Phi}{\partial \bar{\rho}_{i}} \frac{\partial \bar{\rho}_{i}}{\partial \tilde{\rho}_{i}} \frac{\partial \tilde{\rho}_{i}}{\partial \rho_{j}},
$$

where $\frac{\partial \Phi}{\partial \bar{\rho}_{i}}$ is calculated using Eq. (18).

\section{Optimization}

In order to be able to handle multiple optimization objectives (e.g., minimizing the reflectance for frequency $\omega_{1}$ while at the same time minimizing transmittance for $\omega_{2}$ ) the optimization problem is stated as a min/max problem [20,21], where the optimization minimizes the maximum of $i$ th objective functions $h_{i}(\bar{\rho})$ in a given iteration step $n$. In other words the optimization considers the "worst case" of the objective functions, and minimizes with respect to this function in a given iteration step.

If no additional means are taken, optimal designs may be very sensitive to even very small design variations $[\underline{13}, \underline{15}]$. In a practical context we are interested in achieving designs that are robust with respect to manufacturing uncertainties and small variations in the design. A robust optimization procedure based on topology optimization and the projection method has recently been proposed $[19,20]$. This method achieves robust designs by considering a dilated, an intermediate, and an eroded design in the optimization, corresponding to an underetched, normal etched and overetched situation, respectively. By utilizing three different threshold values $\eta, 0.5$, and $1-\eta$ in the projection function, corresponding to a dilated design $\bar{\rho}^{d}$, an intermediate design $\bar{\rho}^{i}$, and a eroded design $\bar{\rho}^{e}$ the optimization scheme can be written as

$$
\begin{gathered}
\min _{\rho_{j}}: \max _{i}\left\{h_{i}\left(\bar{\rho}_{j}^{d}\right), h_{i}\left(\bar{\rho}_{j}^{i}\right), h_{i}\left(\bar{\rho}_{j}^{e}\right)\right\}, \quad \text { s.t.: }\left(\mathbf{K}_{i}^{q}-\omega_{i}^{2} \mathbf{M}_{i}^{q}\right) \mathbf{u}=\mathbf{f}_{i}, \\
0<\rho_{j} \leq 1 \quad i=1, \ldots k, \quad j=1, \ldots N, \quad q=\{e, i, d\} .
\end{gathered}
$$

Each projection in the robust scheme enters the min/max formulation as a separate objective function. Therefore, for each optimization objective of the original problem we have to solve three finite element problems and calculate the design sensitivities for three projections. The blueprint design sent to the manufacturer is the intermediate design. However, the robust optimization ensures that even with quite significant overetching or underetching the resulting structure will perform as well as the blueprint design.

The robust optimization procedure described above ensures robustness with respect to uniform overetching or underetching. Nevertheless, other types of more localized variations may still degrade the performance. Taking all possible variations into account is not practical from the optimization perspective; however, recent research [22] shows that including computationally more intensive studies with localized variations does not change the resulting topologies significantly for static mechanical problems. Whether such a conclusion also holds for the grating problems considered here will be verified in future work.

\section{E. Implementation}

For the finite element analysis 4 noded bilinear rectangular elements are implemented. To ensure a smooth transition between active and passive design domains the filter is allowed to extend into the passive domains and are updated according to the filtered design variables. Symmetry around $y=d / 2$ is enforced and the optimization problem is solved using the deterministic, gradient-based method of moving asymptotes (MMA) [21]. The optimization scheme is outlined as follows.

1. Set the design domain and choose the robust threshold values $\eta^{d}, \eta^{i}$, and $\eta^{e}$.

2. Initialize design variables $\rho$ in the passive and active domains.

3. Filter and project the design variables $\rho$ into the dilated, intermediate, and eroded designs $\rho^{d}, \rho^{i}$, and $\rho^{e}$, respectively.

4. Solve the finite element problem for each optimization objective for each of the three design realizations.

5. Calculate the objectives, constraints and the sensitivities.

6. Update the design variables $\rho$ using MMA.

7. Calculate the maximum change in design variables $\Delta \rho=\max _{i}\left(\left|\Delta \rho_{i}\right|\right)$.

8. For every fortieth iteration or if $\Delta \rho<1 e-2$ and $\beta<\beta_{\max }$, set $\beta=1.355 \beta$.

9. If $\Delta \rho<1 e-2$ and $\beta \geq \beta_{\max }$ terminate, else goto 3 .

The initial value of $\beta$ is 1 and the maximum betacontinuation value is set to $\beta_{\max }=500$. The optimization procedure is implemented in MATLAB and runs in parallel on a standard $2.66 \mathrm{GHz}$ four processor personal computer. All loops have been vectorized for improved speed [23]. The optimizations typically converge in 600-700 iterations and each iteration takes approximately $3 \mathrm{~min}$ for 14 discrete load cases on a $4 \cdot 10^{4}$ degree of freedom problem. For further speed improvement the actual optimization examples are run in 
parallel on the DTU HPC center (www.cc.dtu.dk) on a twoprocessor quad core node decreasing computational time by $66 \%$ on the benchmark example.

\section{NUMERICAL RESULTS}

The described approach is illustrated through the following examples highlighting selected features of the problem. The design domain has dimensions $\{x=1.0 \mu \mathrm{m}, y=1.5 \mu \mathrm{m}\}$ with the active surface region having length $\ell_{D}=0.3 \mu \mathrm{m}$ and the passive areas $\ell_{A}=\ell_{B}=0.35 \mu \mathrm{m}$. A $x=1.6 \mu \mathrm{m}$ wide PML layer is used at both ends. To have a sufficient resolution of the wave in the finite element model the element size is set to $\{0.0125 \mu \mathrm{m} \times 0.0125 \mu \mathrm{m}\}$. If light with a free-space wavelength of $0.6 \mu \mathrm{m}$ travels in plastic with a reflection coefficient of $n=1.5$ the wave is resolved with 32 elements per wavelength. The projection threshold values are chosen as $\left\{\eta^{d}, \eta^{i}, \eta^{e}\right\}=\{0.35,0.50,0.65\}$ and the filter radius is set to $0.1 \mu \mathrm{m}$.

\section{A. Unconstrained Robust Design of Antireflective Surface}

The goal of this example is to maximize the transmission of light at a single wavelength $\lambda_{0}=0.6 \mu \mathrm{m}$ through the plastic interface for an arbitrary input angle. The design domain is initially filled with air $\rho_{i}=0$ except for a single element on the symmetry line, which is given full density and thereby acts as a "seed" for the optimization algorithm avoiding initial problems of local minima of one-dimension designs with constant density in the $y$ direction. The optimization is performed with

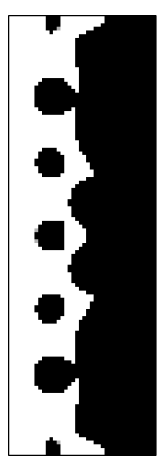

(a)

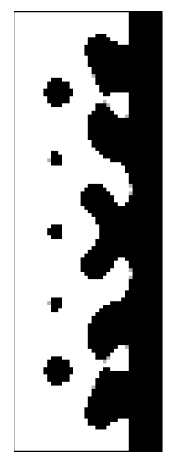

(b)

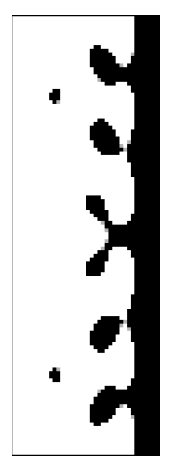

(c)

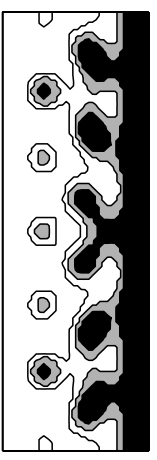

(d)
Fig. 2. Optimized robust design of antireflective surface for seven different input angles. (a) Dilated design, (b) intermediate design, (c) eroded design, and (d) overlaid contour plot comparing the surface contours of the three projections. a given number of wave incident angles as maximization objectives for both $E_{z^{-}}$and $H_{z}$-polarization. Therefore, the total number of objective functions for an optimization using, e.g., four different angles of incidence is 8 .

Figure 2 shows the final optimized robust design using objective functions $h_{i}$ for the input angles $\theta_{i}=$ $\{0,10,20,30,40,50,60\}^{\circ}$ for both polarizations. The dilated, intermediate, and eroded designs are shown together with a contour plot demonstrating how the different threshold values produce different design projections. The contour plot illustrates how variations in, e.g., the size of the manufacturing tool or under-/overetching produce dilated or eroded design realizations which nevertheless all (including intermediate realizations) have the same performances due to the robust design strategy. Figure $\underline{3}$ compares the angle response of optimizations using a varying number of objective angles, and the analytical transmittance of a plane interface is given as reference.

Optimizing using only $0^{\circ}$ angle of incidence as target angle produces optimal performance for this specific incident angle while for higher incident angles the response deteriorates significantly. Including a higher number of input angles improves the response seen across all the objective angles. The final mean transmittance of the objective functions of the intermediate projection is $99.2 \%$ with a standard deviation of $0.31 \%$. To verify that the design is indeed robust toward geometrical variations given by the different projection thresholds the responses of selected angles are plotted in Fig. $\underline{4}$ as a function of the threshold parameter. The plot shows that the response is close to constant in between and even beyond the thresholds values $\eta=0.35-0.65$ indicating that the design is robust and the response is independent of uniform variations in the design realization within the limits of the projection values. For this specific example we found that optimizing for $\mathrm{a} 40^{\circ}$ angle alone or for multiple (seven) angles yields almost the same wide angle performance. However, one cannot expect that this will hold for other examples as illustrated in the next sections, thus motivating the need for the proposed multiobjective approach.

\section{B. Optimization Imposing Mechanical Constraint}

The optimized design in the previous example is not physically connected. For an air/plastic interface this of course makes no physical sense as we require all material be connected to the bulk. In order to ensure a coherent structure a mechanical constraint is imposed the optimization following the procedure from Wang et al. [15]. The isolated components of the
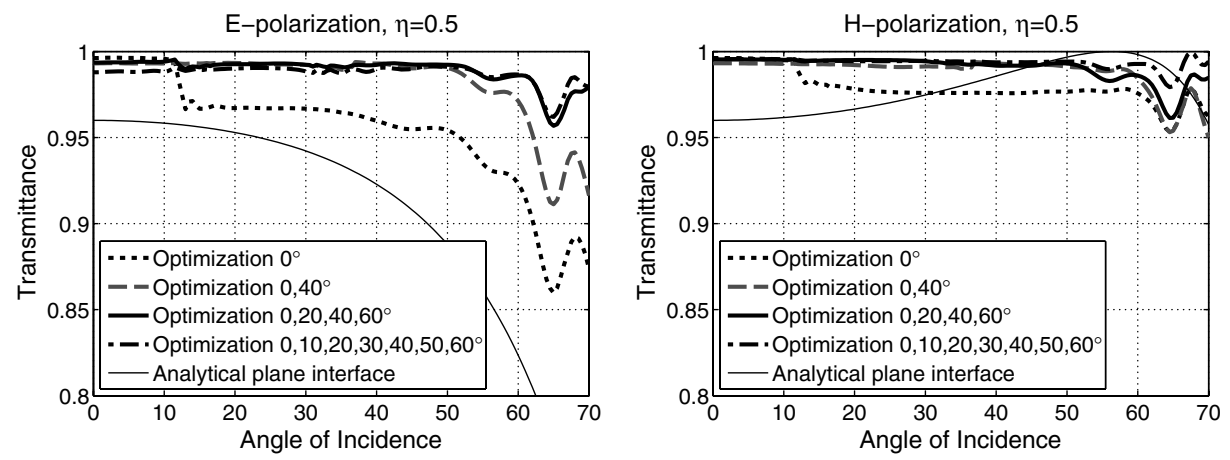

Fig. 3. Angle sweep of robust optimized designs for $\lambda=600 \mathrm{~nm}$ and maximized transmittance. 


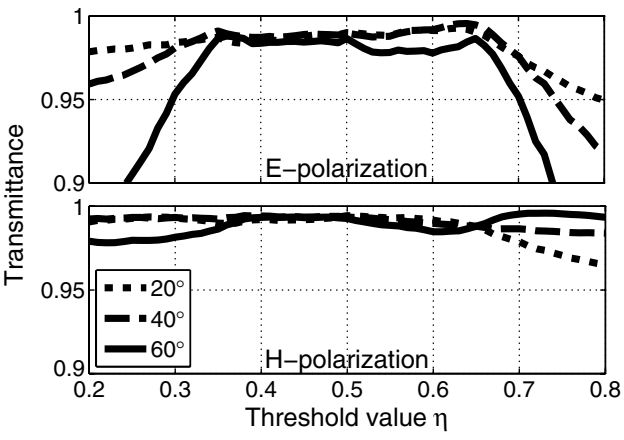

Fig. 4. Threshold value sweep of optimized designs for $\lambda=600 \mathrm{~nm}$ and maximized transmittance.

design cause the fundamental free mechanical vibration frequency of the supercell to be zero. Hence, a constraint on the free mechanical vibration frequency may be added to avoid disconnected material parts. The free mechanical vibration problem can be solved as a mechanical eigenvalue problem of the supercell using the following boundary conditions:

$$
\mathbf{u}(\ell, y)=0, \quad \mathbf{u}(x, 0)=\mathbf{u}(x, d),
$$

where $\ell=\ell_{A}+\ell_{D}+\ell_{B}$ is given in Fig. 1 and the PML layers are discluded from the analysis. The mechanical properties are again interpolated using the solid isotropic material with penalization (SIMP) [12] scheme:

$$
\begin{aligned}
& E_{e}^{q}=E_{\min }+\left(E_{1}-E_{\min }\right)\left(\bar{\rho}_{e}^{q}\right)^{p} \\
& m_{e}^{q}=m_{\min }+\left(m_{1}-m_{\min }\right) \bar{\rho}_{e}^{q} .
\end{aligned}
$$

Here $E_{e}^{q}$ and $m_{e}^{q}$ are the Youngs modulus and mass density of element $e$ with projection $q$, respectively, and $p$ is the exponential penalty of the SIMP model. The parameters are chosen as $E_{1}=10^{3}, m_{1}=1, E_{\min }=10^{-9} E_{1}, m_{\text {min }}=10^{-9} m_{1}$, and $p=5$. The constraint is imposed by requiring that the smallest eigenvalue $\lambda_{1}$ of the system is larger than a given threshold $\delta$. The optimization can now be formulated as

$\min _{\rho_{j}}: \max _{q}: \max _{i}:\left\{h_{i}\left(\left(\tilde{\rho}_{j}\right)^{q}\right)\right\}$,

s.t.: $\left(\mathbf{K}_{i}^{q}-\omega^{2} \mathbf{M}_{i}^{q}\right) \mathbf{u}=\mathbf{f}_{\mathbf{i}}, \quad\left(\hat{\mathbf{K}}_{\mathbf{i}}-\lambda_{1}^{q} \hat{\mathbf{M}}_{\mathbf{i}}\right) x=0, \quad \lambda_{1}^{q}>\delta$,

$0<\rho_{j} \leq 1, \quad i=1, \ldots k, \quad j=1, \ldots N, \quad q=\{e, i, d\}$.

To ensure robustness and efficient implementation of the mechanical constraint, a continuation approach is proposed to gradually increase the threshold value of the mechanical constraint following the increase in the $\beta$ variable:

$$
\delta=\delta_{\min }+\left(\delta_{\max }-\delta_{\min }\right)\left(\frac{\beta-\beta_{0}}{\beta_{\max }-\beta_{0}}\right) .
$$

In Eq. (29), $\delta_{\min }$ and $\delta_{\max }$ are the minimum and maximum values of the mechanical threshold constraint, and $\beta_{0}$ and $\beta_{\max }$ are the initial and maximum values of the $\beta$-continuation parameter. The threshold values are set to $\delta_{\min }=5 e^{-5}$ and $\delta_{\max }=$ $80 e^{-5}$ and it was found that the optimization progressed more smoothly if the initial value of $\beta_{0}$ was put to 8 . The result of the seven angle optimization of the previous example for both $E$-and $H$-polarization with the mechanical constraint is shown in Fig. 5. It is clear that the constraint effectively promotes a coherent design for all three projections. Figure $\underline{6}$ shows an

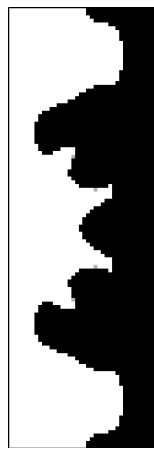

(a)

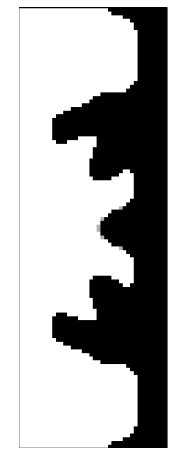

(b)

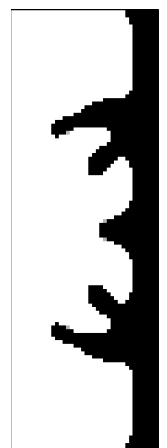

(c)

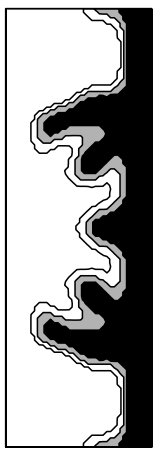

(d)
Fig. 5. Optimized robust design with mechanical constraint for incident angle $[0,10,20,30,40,50,60]^{\circ}$ and $E$ - and $H$-polarized light. (a) Dilated design, (b) intermediate design, (c) eroded design, and (d) overlaid contour plot.

angle sweep of the design, which indicates a slight decrease in performance compared to the nonconstrained examples in Fig. 3. The final mean transmittance of the intermediate projection of the new design is $98.6 \%$ with a standard deviation of $0.4 \%$, which is only slightly worse and with a slightly higher deviation between individual objective functions. It should be noted, that the final design is influenced by the choice of threshold value $\delta$.

\section{Reflective Surface Design for Multiple Frequencies}

The problem of increasing the surface reflection for a range of wavelengths is studied in this example. The objective is to minimize the transmittance for light with free-space wavelength of $\{500,550,600,650,700\} \mathrm{nm}$ and with an incident angle of $40^{\circ}$. Furthermore, the robust projection threshold values were changed to $\eta^{q}=\{0.4,0.5,0.6\}$ decreasing the manufacturing tolerances of the design. In order not to be too restrictive and to give the optimization more freedom, the depth of the design domain is increased to $1.0 \mu \mathrm{m}$. To facilitate convergence in the initial part of the optimization an optimized design without the mechanical constraint was applied as initial guess for the mechanically constrained optimization. Figure 7(a) shows the intermediate projection of the unconstrained optimization and Fig. 7(b) shows the final optimized mechanical constrained design using the unconstrained optimization as initial guess. To maximize reflection the design aligns itself in tilted parallel bands approximately perpendicular to the incident wave angle of $\pm 40^{\circ}$ (symmetry is enforced) and the mechanical constraint ensures connectivity. Figure $\underline{8}$ shows the wavelength sweep of the optimized intermediate design. The sweep clearly shows that the reflectance is significantly increased for the optimized design compared to the reflection from a simple plane interface. It is noted that only the $H$-polarized objective functions are active in the optimization, i.e., it is most challenging to increase the reflection for $H$-polarized light. This corresponds with the higher transmission through a plane interface of $H$-polarized light compared to $E$-polarized of $40^{\circ}$ incident angle (cf. Fig. 3). Although increased, the response varies somewhat in between the chosen wavelength values. To achieve a more uniform response additional intermediate objective wavelengths should be chosen or alternatively one could apply Padé approximations for efficiently calculating responses and sensitivities for a broader frequency range [24]. 


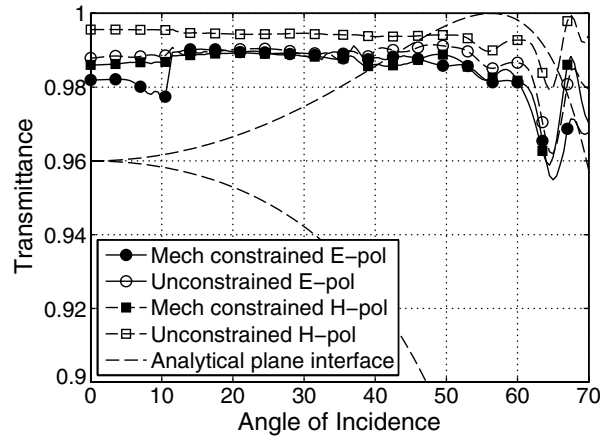

(a)

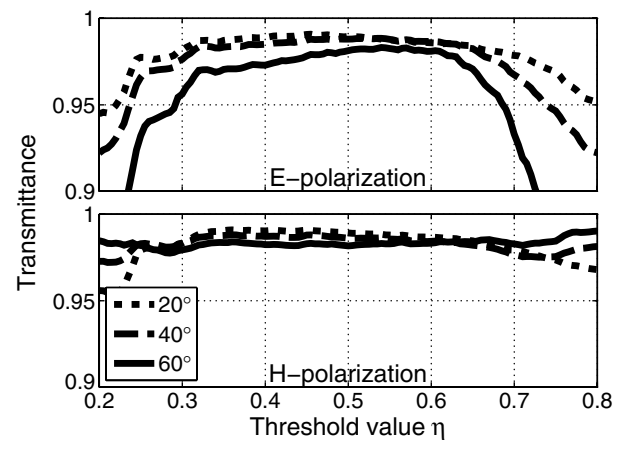

(b)

Fig. 6. Responses for the robust optimization with mechanical constraint for incident angles $[0,10,20,30,40,50,60]^{\circ}$ and $E$ - and $H$-polarized light. (a) Angle sweep of robust optimized intermediate projection design. (b) Threshold value sweep for optimized design.

It must be noted that a perfect design, i.e., zero transmission for all wavelengths for both polarizations obviously can be obtained for a thicker design domain. However, in practice very thick surface structures consisting of multiple layers or inclusions will be very expensive or impractical to manufacture. Hence, we here use the power of the topology optimization approach to work with limited design domain sizes to explore the possibilities of transmission/reflection control within thin surface layers.

\section{Design of Angle Selective Surface}

In this final example the objective is to minimize the transmittance for the $20^{\circ}$ incident angle while maximizing the transmittance for $\{0,10,30,40\}^{\circ}$ incident angles. The design

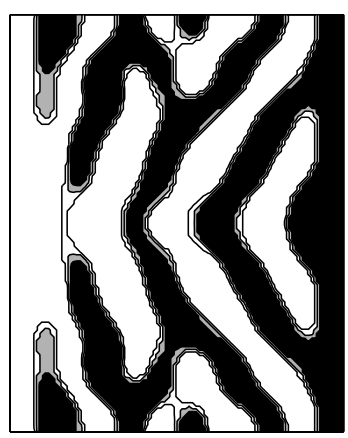

(a)

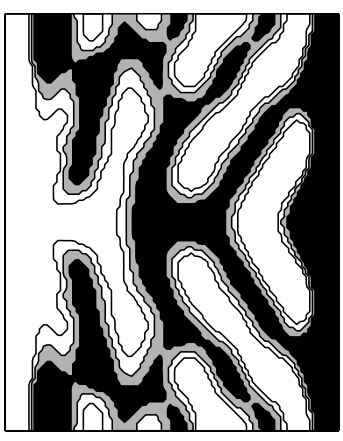

(b)
Fig. 7. (a) Intermediate projection of unconstrained optimization and (b) contour plot of reflective surface design with mechanical constraint using unconstrained optimization design as initial guess.

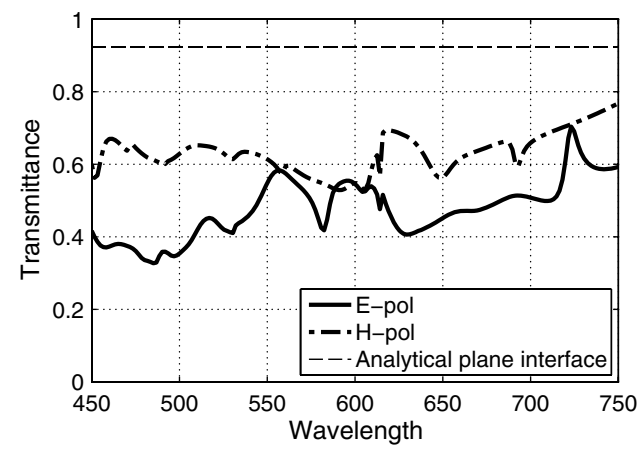

Fig. 8. Wavelength sweep of reflective surface design optimized with objectives of wavelengths [500, 550, 600, 650, 700] nm using unconstrained optimized design as initial guess. therefore has to accommodate multiple objectives with contradictory goals. The design domain depth is $1.0 \mu \mathrm{m}$ and only $E$-polarized light is considered in this example. As in the previous example the optimization requires an initial guess to converge to a solution. Here a simple grating structure of $0.33 \mu \mathrm{m}$ wide and $1 \mu \mathrm{m}$ deep square trenches are used as initial guess. The resulting contour plot of the robust design is shown in Fig. 9, the angle sweep in Fig. 10, and the realized microstructure constituted by four periods is illustrated in Fig. 11 . As can be seen from the angle sweep, the transmittance is significantly decreased for $20^{\circ}$ and remains relatively high for the adjacent incident angles. Angles above $40^{\circ}$ are not considered in the optimization. As can be seen the maximum transmittance is now significantly lower ( $70 \%-80 \%$ transmittance) than example $\underline{4 . \mathrm{B}}$ because the design has to accommodate the minimum reflection for the $20^{\circ}$ incident angle.

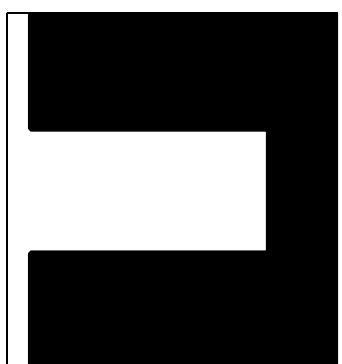

(a)

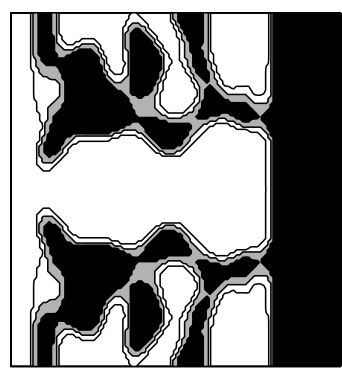

(b)
Fig. 9. (a) Initial guess and (b) contour plot of optimized angle selective surface design.

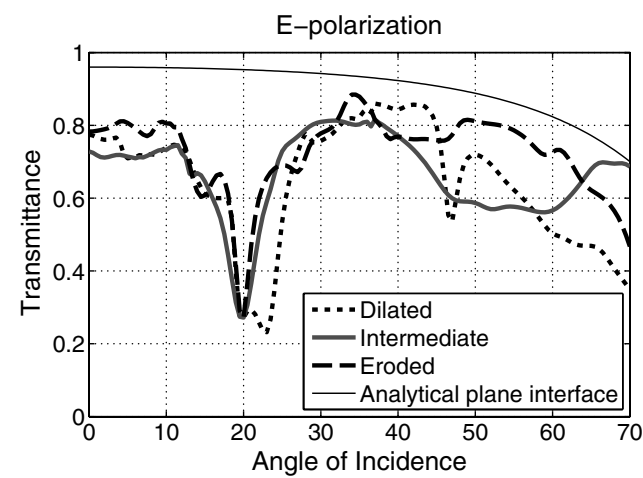

Fig. 10. Angular sweep for optimized angle selective surface design. 


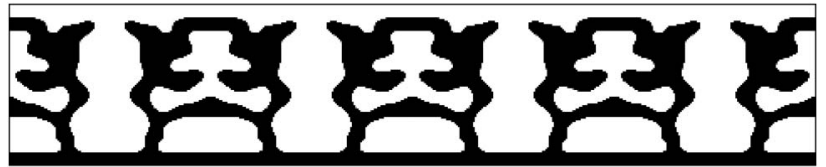

Fig. 11. Surface microstructure constituted by optimized intermediate angle selective surface design.

\section{DISCUSSION}

The proposed method shows how topology optimization can be used to design optical gratings with tailored-angle and frequency-dependent reflectance properties. Although all examples include air/plastic interfaces, the method is applicable to any optical material interface. The inclusion of damping is trivial. Hence, it can be used in many applications for designing surface gratings with advanced responses and robustness toward manufacturing errors. In relation to the ambition of creating structural color from the tailored microstructure in plastic surfaces the proposed optimization scheme could be utilized to promote the reflection of specific wavelengths. As was indicated by Lee and Smith [5] the structural colors of butterflies can be explained by the small variation in height and tilt of the individual periods of the microstructure of the wings which contribute to a constant color sensation across a broad interval of viewing angles. This implies that the analysis of strictly periodic structures could be used for designing surfaces for achieving structural colors in a broad spectrum of viewing angles.

As shown in the examples and observed by Fuchi [11] optimal reflective material design often consists of multiple unconnected parallel bands that may be unsuited for surface manufacturing. The mechanical constraint effectively ensures a connected design; however, there is no constraint on the formation of closed pockets inside the structure nor large overhangs. The angle selective reflection structure in Fig. 11 may therefore be a challenge from a manufacturing point of view. Additional manufacturing constraints relevant for the specific manufacturing process could be formulated, however a poorer performance of the design is expected due to decreased design freedom.

Achieving minimum length scale and mesh independence in topology optimization has long been a challenge. An overview of the development and solutions to these problems are given by Wang et al. [19]. The robust formulation used in the present work provides local minimum length scale control as long as the overall topology of the three projected designs is the same. The fundamental free mechanical vibration frequency constraint ensures the same topology for all projections and hence ensures a local minimum length scale in the design. The minimum length scale can be controlled by selecting appropriate combinations of the filter radius and projection threshold values.

\section{CONCLUSION}

In this study a general method based on robust topology optimization for designing periodic surfaces with tailored optical properties has been presented. The method is highly versatile and can be used for designing surface grating with extreme reflectance or transmittance characteristics and/or advanced frequency and angle-dependent responses. Numerical examples show the flexibility of the $\mathrm{min} / \mathrm{max}$ formulation in formulating complex design optimization objectives. Differing from previous studies using topology optimization for designing periodic grating like structures the present formulation ensures final black and white designs by a Heaviside projection scheme. A robust formulation has been implemented to partly ensure design tolerances toward uncertainties in realizing the design, and partly to ensure minimum length scale on the design. It was found, that in order to ensure a coherent and physical sound design a mechanical constraint based on the fundamental free mechanical vibration frequency should be used.

\section{ACKNOWLEDGMENTS}

We appreciate the support from the Danish National Advanced Technology Foundation through the project NANOPlast, the Villum Foundation through the NextTop project, and the Danish Center for Scientific Computing (DCSC).

\section{REFERENCES}

1. L. D. Chiffre, H. Kunzmann, G. N. Peggs, and D. A. Lucca, "Surfaces in precision engineering, microengineering and nanotechnology," CIRP Ann. 52, 561-577 (2003).

2. L. Alting, F. Kimura, H. N. Hansen, and G. Bissacco, "Micro engineering," CIRP Ann. 52, 635-657 (2003).

3. P. Utko, F. Persson, A. Kristensen, and N. B. Larsen, "Injection molded nanofluidic chips: fabrication method and functional tests using single-molecule DNA experiments," Lab Chip 11, 303-308 (2011).

4. K. O. Andresen, M. Hansen, M. Matschuk, S. T. Jepsen, H. S. Sorensen, P. Utko, D. Selmeczi, T. S. Hansen, N. B. Larsen, N. Rozlosnik, and R. Taboryski, "Injection molded chips with integrated conducting polymer electrodes for electroporation of cells," J. Micromech. Microeng. 20, 055010 (2010).

5. R. T. Lee and G. S. Smith, "Detailed electromagnetic simulation for the structural color of butterfly wings," Appl. Opt. 48, 4177-4190 (2009).

6. S. Banerjee, J. B. Cole, and T. Yatagai, "Colour characterization of a morpho butterfly wing-scale using a high accuracy nonstandard finite-difference time-domain method," Micron 38, 97-103 (2007).

7. K. Chung, S. Yu, C.-J. Heo, J. W. Shim, S.-M. Yang, M. G. Han, H.-S. Lee, Y. Jin, S. Y. Lee, N. Park, and J. H. Shin, "Flexible, angle-independent, structural color reflectors inspired by morpho butterfly wings," Adv. Mater. 24, 2375-2379 (2012).

8. J. S. Jensen and O. Sigmund, "Topology optimization for nanophotonics," Laser Photon. Rev. 5, 308-321 (2011).

9. D. C. Dobson, "Optimal design of periodic antireflective structures for the Helmholtz equation,” Eur. J. Appl. Math. 4, 321-339 (1993).

10. D. C. Dobson, "Optimal shape design of blazed diffraction gratings,” Appl. Math. Optim. 40, 61-78 (1999).

11. K. Fuchi, A. R. Diaz, E. Rothwell, R. Ouedraogo, and A. Temme, "Topology optimization of periodic layouts of dielectric materials," Struct. Multidisc. Optim. 42, CP11-493 (2010).

12. M. P. Bendsoe and O. Sigmund, Topology OptimisationTheory, Methods and Applications, 2nd ed. (Springer-Verlag, 2003).

13. J. S. Jensen and O. Sigmund, “Topology optimization of photonic crystal structures: a high-bandwidth low-loss $t$-junction waveguide," J. Opt. Soc. Am. B 22, 1191-1198 (2005).

14. J. Jin, The Finite Element Method in Electromagnetics, 2nd ed. (Wiley-IEEE, 2002).

15. F. Wang, J. S. Jensen, and O. Sigmund, "Robust topology optimization of photonic crystal waveguides with tailored dispersion properties,” J. Opt. Soc. Am. B 28, 387-397 (2011).

16. J. S. Jensen, "A note on sensitivity analysis of linear dynamic systems with harmonic excitation," Tech. rep. (Technical University of Denmark, Department of Mechanical Engineering, 2009). 
17. O. Sigmund, "Morphology-based black and white filters for topology optimization," Struct. Multidisc. Optim. 33, 401-424 (2007).

18. J. Guest, J. Prevost, and T. Belytschko, "Achieving minimum length scale in topology optimization using nodal design variables and projection functions," Int. J. Num. Methods Eng. 61, 238-254 (2004).

19. F. Wang, B. S. Lazarov, and O. Sigmund, "On projection methods, convergence and robust formulations in topology optimization," Struct. Multidisc. Optim. 43, 767-784 (2011).

20. O. Sigmund, "Manufacturing tolerant topology optimization," Acta Mech. Sin. 25, 227-239 (2009).
21. K. Svanberg, "Method of moving asymptotes-a new method for structural optimization,” Int. J. Num. Methods Eng. 24, 359-373 (1987).

22. M. Schevenels, B. S. Lazarov, and O. Sigmund, "Robust topology optimization accounting for spatially varying manufacturing errors," Comput. Methods Appl. Mech. Eng. 200, 3613-3627 (2011).

23. E. Andreassen, A. Clausen, M. Schevenels, B. S. Lazarov, and O. Sigmund, "Efficient topology optimization in MATLAB using 88 lines of code," Struct. Multidisc. Optim. 43, 1-16 (2011).

24. J. S. Jensen, "Topology optimization of dynamics problems with Padé approximants," Int. J. Num. Methods Eng. 72, 1605-1630 (2007). 